\title{
Mechanical deformation of InP and GaAs by spherical indentation
}

\author{
J. E. Bradby, ${ }^{\text {a) }}$ J. S. Williams, and J. Wong-Leung \\ Department of Electronic Materials Engineering, Research School of Physical Sciences and Engineering, \\ The Australian National University, Canberra, ACT 0200, Australia \\ M. V. Swain \\ Biomaterials Science Research Unit, Department of Mechanical and Mechatronic Engineering and Faculty \\ of Dentistry, The University of Sydney, Eveleigh, NSW 1430, Australia \\ P. Munroe \\ Electron Microscope Unit, University of New South Wales, Sydney, NSW 2052, Australia
}

(Received 20 October 2000; accepted for publication 23 March 2001)

\begin{abstract}
The mechanical deformation by spherical indentation of both crystalline InP and GaAs was characterized using cross-sectional transmission electron microscopy (XTEM) and atomic force microscopy. All load-unload curves show a discontinuity (or "pop in") during loading. Slip bands oriented along $\{111\}$ planes are visible in XTEM micrographs from residual indentations in both materials and no evidence of any phase transformations was found. Higher load indentations (35 $\mathrm{mN}$ for $\mathrm{InP}$ and $50 \mathrm{mN}$ for $\mathrm{GaAs}$ ) also revealed subsurface cracking. In contrast no cracking was found beneath a $25 \mathrm{mN} \mathrm{InP}$ indent although the hardness and modulus data are almost identical to those of the cracked sample. The subsurface cracks are thought to be nucleated by high stress concentrations caused by dislocation pileup. (C) 2001 American Institute of Physics.
\end{abstract}

[DOI: $10.1063 / 1.1372207]$

The mechanical deformation responses of semiconductor materials to contact-induced damage is an area of continuing technological interest. ${ }^{1}$ Previous work has shown characteristic discontinuities upon loading ("pop in") for silicon and compound semiconductors. ${ }^{2-4}$ However, silicon seems unique in exhibiting similar discontinuities ("pop out") when unloading. Phase changes induced by indentation loading have been identified in silicon indentations by transmission electron microscopy (TEM) and Raman microspectroscopy. ${ }^{5,6}$ Upon hydrostatic loading, diamond cubic $\mathrm{Si}-\mathrm{I}$ is known to transform to the metallic $\beta$-Sn phase, Si-II. ${ }^{7}$ Upon rapid pressure release, amorphous silicon is formed, but a mixture of the high pressure polymorphs Si-III and $\mathrm{Si}$-XII occurs on slow pressure release indentations. ${ }^{6}$ The interpretation of pop-in and pop-out events for Si has been explained in terms of phase transformations. ${ }^{8}$ It has recently been suggested that this pop-in discontinuity upon loading could be caused by the expansion, beyond the diameter of contact, of transformed material initiated under the indenter; this results in the sudden flow of material around the indenter. ${ }^{9}$

The onset of plastic deformation, denoted by the pop in, is a catastrophic event in compound semiconductors such as GaAs, InP and GaN. Williams et al. found the contact pressure (or Meyers hardness) can increase to almost twice the accepted value before abruptly falling and remaining at a constant level with increasing indenter penetration. ${ }^{10}$ Slip has been proposed as the mechanism for plastic deformation in compound semiconductors with Page et al. showing slip steps on the face of indentation impressions. ${ }^{11}$ The preferential slip planes for the face-centered-cubic structures of $\mathrm{Si}$, InP and GaAs are $\{111\}$.

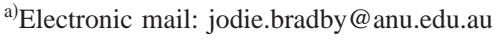

To better understand the mechanism of plastic deformation of compound semiconductors, in this letter we focus on the indentation-induced pop in and cracking of $\mathrm{InP}$ and GaAs. A series of indentations were made in crystalline (100) $\mathrm{InP}$ and GaAs at loads of up to $50 \mathrm{mN}$. The indentation device used was the Ultra-Micro indentation system-2000 (UMIS) with a spherical indenter of $\sim 4.2 \mu \mathrm{m}$ radius. The UMIS and indenter tip were carefully calibrated using fused silica of known material properties. A load-unload cycle was used to create the series of indents for cross-sectional analysis. The partial load-unload cycle (unload to 50\%) was used to extract hardness and elastic modulus as a function of indenter penetration below contact. ${ }^{12}$ The contact diameter of the tip was also calculated as a function of depth of penetration. A Nanoscope III atomic force microscope was used in contact mode to image a series of residual indent impressions in InP at 25 and $35 \mathrm{mN}$ and in GaAs at $50 \mathrm{mN}$. All indentations were made at ambient pressure and room temperature.

Cross-sectional transmission electron microscopy (XTEM) samples were prepared from 25 and $35 \mathrm{mN}$ indents in $\mathrm{InP}$ and $50 \mathrm{mN}$ indents in GaAs. These samples were mechanically polished with the final thinning to electron transparency achieved using an FEI $\times$ P200 focused ion beam (FIB) system. This technique has been used previously to create XTEM samples of indentations in silicon. ${ }^{9,13}$ To protect the surface of the samples during the ion milling process a $\sim 2 \mu \mathrm{m}$ thick layer of platinum was deposited over the surface of the indents using the FIB instrument. The TEM used in this study was a Philips EM 430 operated at an accelerating voltage of $300 \mathrm{kV}$.

The discontinuity or pop in during loading can be clearly seen in the typical load-unload curves shown in Figs. 1(a) and 1(b) for InP and GaAs, respectively. The pop in for InP, 


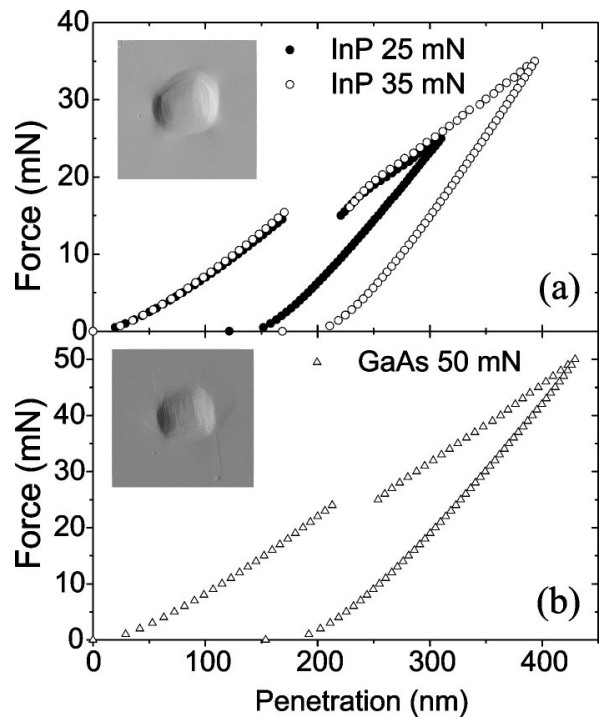

FIG. 1. Typical continuous load-unload curves of (a) InP with maximum loads of 25 and $35 \mathrm{mN}$. Inset: An AFM image of an indent in InP with a maximum load of $35 \mathrm{mN}$ (field width $7 \mu \mathrm{m}$ ) and (b) GaAs with a maximum load of $50 \mathrm{mN}$. Inset: An AFM image of an indent in GaAs with a maximum load of $50 \mathrm{mN}$ (field width $7 \mu \mathrm{m}$ ).

shown in Fig. 1(a), occurs at a load of $\sim 15 \mathrm{mN}$. The pop-in load was found to vary between 10 and $17 \mathrm{mN}$. The same loading pattern is seen for indents made in InP at maximum loads of 25 and $35 \mathrm{mN}$. The inset in Fig. 1 shows a contact mode atomic force microscopy (AFM) image (field width 7 $\mu \mathrm{m})$ of a $35 \mathrm{mN}$ indent in InP with no visible surface cracking. Section analysis of the AFM data revealed pileup of $\sim 15 \mathrm{~nm}$ occurring in four lobes at $90^{\circ}$ intervals around the periphery of the residual indent impression. A spread of pop-in loads is also observed for GaAs varying between 23 and $45 \mathrm{mN}$, with Fig. 1(b) showing a typical loading curve to a maximum load of $50 \mathrm{mN}$. The inset shows a contact mode AFM image (field width $7 \mu \mathrm{m}$ ) of a $50 \mathrm{mN}$ indent in GaAs that illustrates slip lines within the residual indent impression. Pileup in four lobes was also seen from section analysis of the AFM data of the residual indent impression, similar to that found around the InP indent.

The hardness of InP and GaAs as a function of depth of indenter penetration below contact ${ }^{12}$ is shown in Fig. 2(a). Both materials show the onset of plastic deformation to be a catastrophic event. The hardness after pop in for InP is $\sim 5.1$ and $\sim 7.5 \mathrm{GPa}$ for GaAs. InP indented with maximum loads of 25 and $35 \mathrm{mN}$ shows almost identically shaped curves. The Young's modulus shown in Fig. 2(b) as a function of penetration depth below contact (underestimation of the absolute modulus due to the effect of indenter compliance) reveals no significant differences between the two maximum loading conditions in InP. The gap in the data points, resulting from a sudden increase in depth, is characteristic of the pop-in event. The value of the modulus is not affected by the pop in and remains relatively constant at $\sim 82 \mathrm{GPa}$ for InP and $\sim 97 \mathrm{GPa}$ for GaAs. The average contact diameter of the indenter tip at maximum depth for both $\mathrm{InP}$ at $35 \mathrm{mN}$ and GaAs at $50 \mathrm{mN}$ was found to be $\sim 2.9 \mu \mathrm{m}$.

Shown in Figs. 3(a)-3(c) are XTEM bright field micrographs of indents made in InP at maximum loads of 25 and $35 \mathrm{mN}$ and in GaAs at $50 \mathrm{mN}$, respectively. The uneven

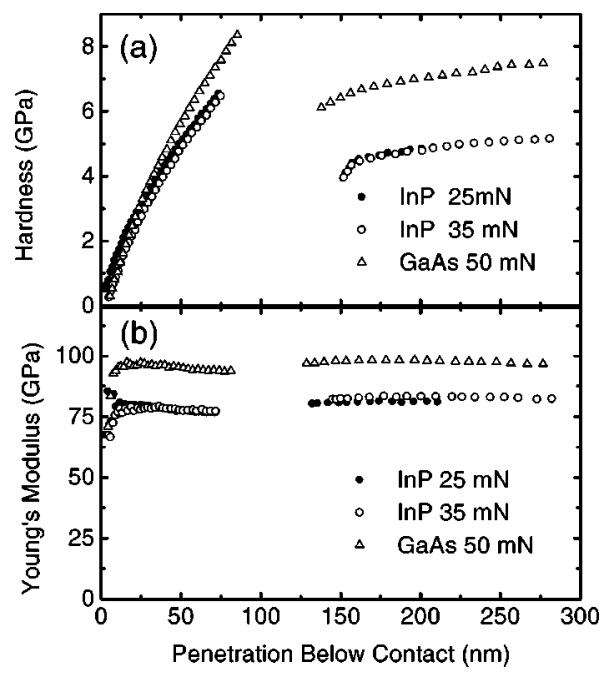

FIG. 2. Curves of (a) the hardness and (b) Young's modulus as a function of indenter penetration below contact for InP at maximum loads of 25 and 35 $\mathrm{mN}$ and $\mathrm{GaAs}$ at a maximum load of $50 \mathrm{mN}$.

nature of the surface and the slightly mottled appearance of the surrounding InP are thought to be caused during the FIB milling process. Slip bands along the $\{111\}$ can be clearly seen in all micrographs. The slip bands are oriented parallel along two planes, both aligned at an angle of $54^{\circ}$ to the (100) surface. The maximum diameters of the slip bands at the surface of the $\mathrm{InP}$ indents are $\sim 1.4$ and $\sim 3.4 \mu \mathrm{m}$ for the 25
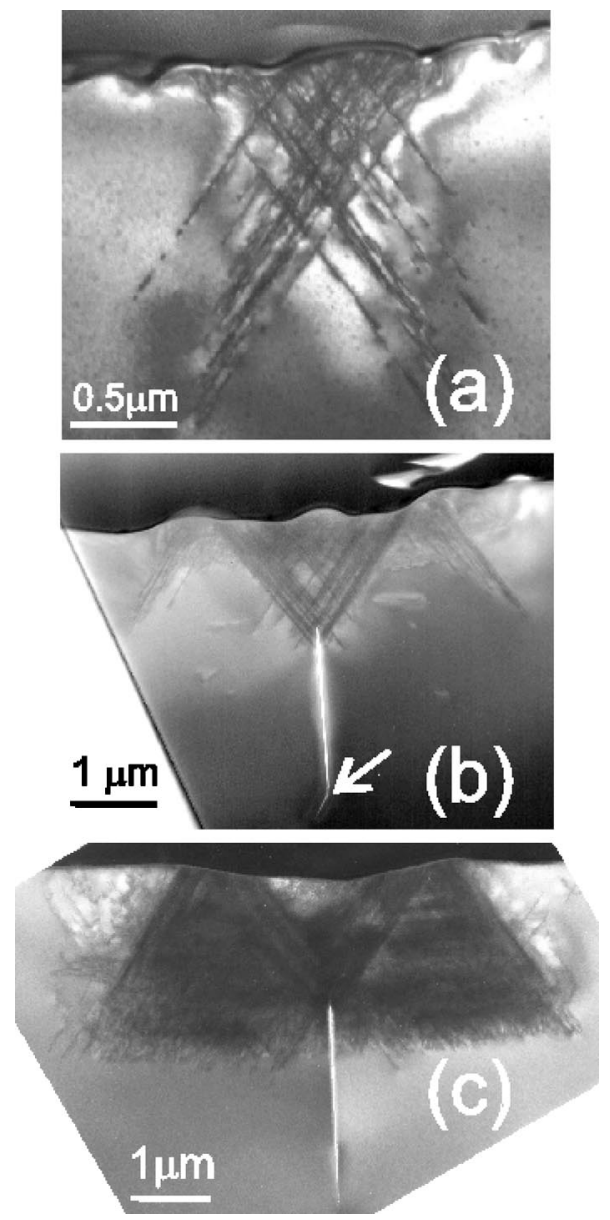

FIG. 3. XTEM zone axis bright field images of indents formed in (a) InP at a maximum load of $25 \mathrm{mN}$, (b) InP at a maximum load of $35 \mathrm{mN}$ (the arrow denotes the change in direction of the crack) and (c) GaAs at a maximum load of $50 \mathrm{mN}$. 
and $35 \mathrm{mN}$ indents, respectively. The apex of the bands is $\sim 1.1 \mu \mathrm{m}$ from the surface for the lower-load InP indent and $\sim 1.5 \mu \mathrm{m}$ for the higher-load InP indent. The diameter of the slip band region at the surface of the GaAs indent is $\sim 3.1 \mu \mathrm{m}$ and extends to a depth of $\sim 1.6 \mu \mathrm{m}$. A selected area diffraction study was carried out in these samples and no evidence of any phase transformation was found.

In addition to the slip bands, a median crack (crack direction $\langle 001\rangle)$ under the residual indent impression is shown in Figs. 3(b) and 3(c). The crack seems to be nucleated near the apex of the slip bands in both cases with the widest section of the crack $(\sim 57 \mathrm{~nm}$ for $\mathrm{InP}$ at $35 \mathrm{mN}$ and $\sim 42 \mathrm{~nm}$ for GaAs at $50 \mathrm{mN}$ ) located in this region. The crack extends down into the bulk of the crystal and, for the $35 \mathrm{mN} \mathrm{InP}$ indent shown in Fig. 3(b), changes in direction of the crack occur as it nears its maximum extension (denoted by an arrow in Fig. 3). It is reasonable to assume that, immediately after crack nucleation, the energetic crack tip progresses straight down into the unstrained crystal. As the energy of the tip is dispersed its direction can then be influenced by the crystal structure of the material.

The nucleation of the median crack beneath the slip band apex indicates increased stress concentrations in this region. This stress concentration is likely to be caused by dislocation pileup as the slip bands intersect and act as respective barriers obstructing further dislocation glide. This dislocation pileup can concentrate shear stresses at the slip band extremity and thus cause the nucleation of a microcrack. ${ }^{14}$

The formation of a median crack beneath the indents in both InP and GaAs is not associated with any significant discontinuity in the load-unload data. This is clearly indicated by the similarity in the data collected from the lowerload InP indent (with no crack) and the higher-load indent (with a crack). The pop-in event occurs for both loads and hence cannot be attributed to the sudden failure by cracking of the material.

In contrast to $\mathrm{Si}$, these results verify slip as the mechanism of plastic deformation by spherical indentation for both InP and GaAs. The discontinuity upon loading (pop in) is likely to be caused by the sudden nucleation of slip by the indenter. The observed spread in the pop-in loads for InP and GaAs is consistent with the paucity of defects in these near perfect single crystalline materials and hence the difficulty of nucleating slip. The contact-induced shear stresses for a sphere in contact with a flat surface are at a maximum on the axis of contact at a depth of approximately half the contact radius. ${ }^{15}$ This is indeed the region where many of the slip bands in InP [Fig. 3(b)] intersect. As the loading continues, an increasing number of dislocations glide down the slip bands resulting in dislocation pileup as they intersect. This causes a median crack to become nucleated to relieve these localized stress concentrations within the weak tensile stress field that exists below the hydrostatic compressive zone directly beneath the indenter. A further contributing factor for the pop in might be the formation of slip bands beyond the diameter of constraint of the indenter. Such nonconstrained slip bands may pop out at the surface beyond the contact diameter, causing the four lobe pileup observed in the AFM analysis.

In conclusion, we have shown that the initial response of InP and GaAs to mechanical deformation by spherical indentation is the formation of slip bands, with no phase transformation being observed. Upon further loading, a median crack appears beneath the indent, initiated by dislocation pileup. No differences were found in the hardness and modulus data between the intact and cracked InP samples. This indicates the formation of the median crack does not significantly alter the mechanical properties of the material.

${ }^{1}$ R. G. Jasinevicius, P. S. Pizani, and J. G. Duduch, J. Mater. Res. 15, 1688 (2000).

${ }^{2}$ E. R. Weppelmann, J. S. Field, and M. V. Swain, J. Mater. Sci. 30, 2455 (1995).

${ }^{3}$ T. Page, W. C. Oliver, and C. J. McHargue, J. Mater. Res. 7, 450 (1992).

${ }^{4}$ S. O. Kucheyev, J. E. Bradby, J. S. Williams, C. Jagadish, M. Toth, M. R. Phillips, and M. V. Swain, Appl. Phys. Lett. 77, 3373 (2000).

${ }^{5}$ A. B. Mann, D. van Heerden, J. B. Pethica, and T. P. Weihs, J. Mater. Res. 15, 1754 (2000).

${ }^{6}$ A. Kailer, Y. G. Gogotsi, and K. G. Nickel, J. Appl. Phys. 81, 3057 (1997).

${ }^{7}$ J. Z. Hu, L. D. Merkle, C. S. Menoni, and I. L. Spain, Phys. Rev. B 34, 4679 (1986).

${ }^{8}$ J. J. Gilman, J. Mater. Res. 7, 535 (1992).

${ }^{9}$ J. E. Bradby, J. S. Williams, J. Wong-Leung, M. V. Swain, and P. Munroe, Appl. Phys. Lett. 77, 3749 (2000).

${ }^{10}$ J. S. Williams, Y. Chen, J. Wong-Leung, A. Kerr, and M. V. Swain, J. Mater. Res. 14, 2338 (1999).

${ }^{11}$ T. F. Page, L. Riester, and S. V. Hainsworth, Mater. Res. Soc. Symp. Proc. 522, 113 (1998).

${ }^{12}$ J. S. Field and M. V. Swain, J. Mater. Res. 8, 297 (1993).

${ }^{13}$ H. Saka, J. Vac. Sci. Technol. B 16, 2522 (1998).

${ }^{14}$ A. N. Stroh, Proc. R. Soc. London, Ser. A 223, 404 (1954).

${ }^{15}$ B. Lawn, Fracture of Brittle Solids (Cambridge University Press, Cambridge, 1993). 